\title{
Embedded-based Instrumentation System for Electrical Resistivity Measurement on Conductive Concrete using Four-Electrode Method
}

\author{
La Ode Husein Z Toresano \\ Department of Physics (System and Physics Instrumentation Division), \\ Laboratory of Instrumentation, \\ University of Indonesia, Kampus UI, Depok, 16424, Indonesia \\ E-mail:laode.husein@sci.ui.ac.id \\ Prawito \\ Department of Physics (System and Physics Instrumentation Division), \\ Laboratory of Microcontroller/Microprocessor (Embedded System), \\ University of Indonesia, Kampus UI, Depok, 16424, Indonesia \\ E-mail: prawito@sci.ui.ac.id \\ Arief Sudarmaji \\ Multiferroic Research Group (Material Science), Laboratory of Multiferroic, \\ Department of Physics, University of Indonesia \\ Kampus UI, Depok, 16424, Indonesia
}

\begin{abstract}
Instrumentation system for testing of conductive concrete material using four-electrode method has been built. The system can measure material resistivity under the influence of voltage-injection variable, temperature changing in the characterization reactor, as well as the duration of the material characterization. The system uses an ACS-172 electrical current sensor, a DS-1820 temperature sensor to measure the temperature of the material, a SHT-11 sensor to measure the temperature and humidity of the material characterization reactor, and a DS-1307 RTC (RealTime Clock) system for timing system and reference for controlling the duration of the material testing. The standard method of instrument for measure has been developed based on four electrode method. The system of instrumentation can be measured to numerical value of electrical conductivity and resistivity on material. This instrumentation system which has a heating reactor for the material sample, uses AVR-ATmega-128 as the controller element, and also has an computer interface to connect with LabVIEW-National Instrument. This system can be operated in dual-mode, that is automatic and manual mode. The automatic mode can be generated standard configuration based on computer connection (this part can be displayed a numerical data and real-time graph, and also has to recording/saving a numerical data), whereas manual mode only displayed of data without computer connection. The system of instrument has been developed to measure of electrical resistivity/conductivity, temperature and humidity in reactor, electrical current injected, voltage injection and voltage load on sample, and also setting of real-time date/time for automatic control process of characterization with variation of voltage injection, temperature/humidity, and time for material characterization process.
\end{abstract}

Keywords: electrical resistivity, four-electrode, conductive-concrete, instrumentation, embedded, microcontroller

\section{Introduction}

This study focuses on the design and implementation of embedded-based instrumentation system for measuring the electrical resistivity of a conductive concrete material. The purpose of this instrument is to simplify the process of electrical characterization of the conductive concrete material. This instrument will perform the characterization process by providing a variation of electrical potential difference injected to the sample, a variation of the heating reactor temperature, as well as a variation of the duration of the characterization process. The overall in this process is 
controlled by a microcontroller which can be set in two modes (automatic and manual modes).

A research on a such similar instrumentation system has been conducted. The system is known as iTHERMST, commonly called the Intelligent Remotelycontrolled Thermostat with data logging ${ }^{1}$. iTHERMST was designed and built to test and measure samples of concrete. It can support academic researcher to find proper admixture ratio and pattern that is suitable for a fabrication of heating elements. It is used to test fabrication concrete blocks with dimension of $2 \times 1 \mathrm{~m}$ that was able to melt snow away from the surface effectively ${ }^{1}$.

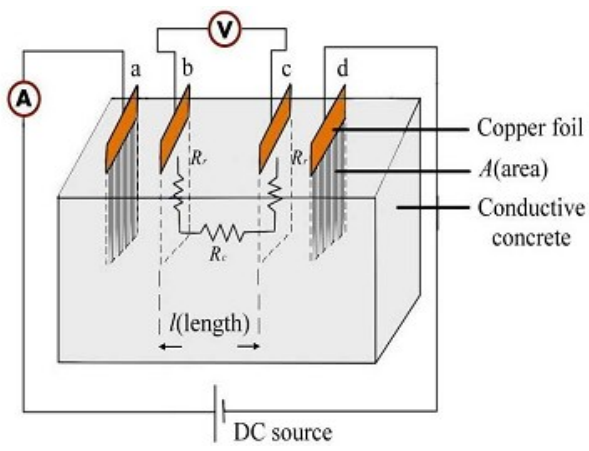

Fig. 1. Configuration of Four Electrode Method for Measuring Electrical Resistivity ${ }^{5}$

In the future, this embedded-based instrumentation system is expected can be applied not only to characterize the resistivity of a conductive material, but also to can be used as an analyzer system to determine the corrosion rate and crack of a material. Moreover, it can be equipped with a set of greater numbers of electrodes in order to develop a electrical resistivitybased tomography instrumentation system. Potential applications of Electrical Resistance Tomography (ERT) in the construction industry are include measurement of the thickness of the concrete cover on top of reinforcement bars location provided by ERT could be utilized in diagnosing the corrosion rate of reinforcement bars ${ }^{2}$. The other researches that have been carried-out 3,4 , show that electrical resistivity measurement can be applied to determine crackings on reinforced concrete structures and also to evaluate the corrosion of reinforcing steel.

\section{Embedded-based Instrumentation System}

The instrumentation system has been implemented using four electrode method for measuring the electrical

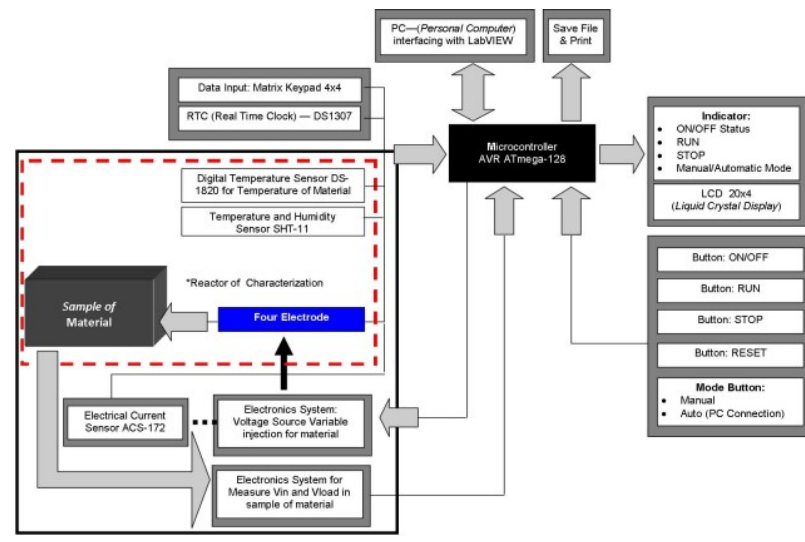

Fig. 2. Block Diagram of Embedded-based Instrumentation System.

resistivity of the conductive concrete materials. The following fig. 1 is the basic configuration four-electrode method that is used to measure the electrical resistivity of the materials.

Fig. 2 is the block diagram of the system that has been designed using a AVR Atmega-128 microcontroller as the core of the system. Fundamentally, it consists of the following parts:

- Power supply unit for overall electronics system.

- Power supply unit as a voltage source injected to the sample.

- Microcontroller system.

- Sensor and their electronics of signal conditioning.

- $\quad$ Panel for Operational Control.

The DC (Direct Current) Power Supply unit for overall supporting electronic system has 5 volts DC voltage (voltage regulated) and a current capacity, while the voltage source unit (for electrical injection for material) is a PWM-based controllable power supply unit has a capacity of 44 Volt a maximum. This unit can generate a voltage value as requested by the user through a keypad or by a microcontroller. The panels of casing are part of the instrumentation system consisting of such activation panel: ON/OFF, MODE button (AUTO - with computer connection and MANUAL data only displayed on the LCD). The RUN button use to activation a system of instrumentation, and STOP button for disable to running of instrument. Additional button, a RESET button has been connected from a reset channel of microcontroller, and the system to reset 
function for stop to running system, if a trouble of running in embedded system of instrumentation.

The process of inputting the manual mode works by inserting the value of a parameter characterizing the

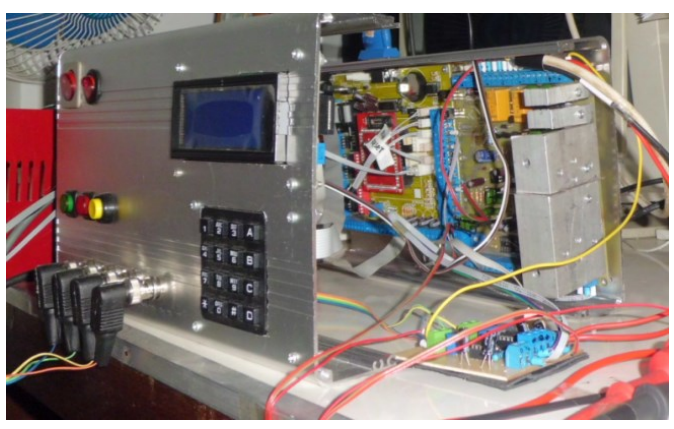

Fig. 3. Instrumentation System of Conductive Concrete material based embedded systems to Electrical Resistivity Characterization.

electrical voltage injection, temperature, and time. The configuration is done via the $4 \times 4$ keypad and displayed to LCD 20x4. LCD (Liquid Crystal Display) is the appearance of numeric data results in the characterization process. On the panel, there is a connection system for input channel of temperature sensor, temperature/humidity sensor, electrode/probe connector, as well as other additional of button in panel.

Fig. 3, elements contained in the Embedded System based AVR ATmega-128:

- Module ATmega-128+ISP - IE.

- Electrical Current Sensors ACS-172.

- Digital Temperature Sensor DS-1820.

- Temperature \& Humidity Sensor SHT-11.

- RTC (Real Time Clock) DS-1370.

- Serial Communication with MAX RS-232 Serial Communication Port.

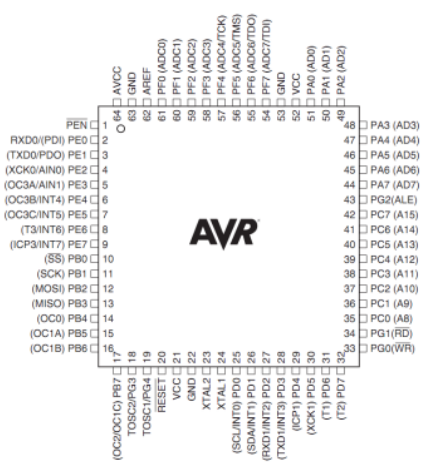

Fig. 4. Pin configuratioin on chip Atmel®AVR ${ }^{\circledR}$ ATmega 128 microcontroller ${ }^{6}$
Additional connectors on embedded systems:

- ADC (Analog to Digital Converter) 14-Bit MAX 194.

- $\mathrm{MMC}$ (Memory Storage) Connection Panel.

AVR Atmega-128 is a family member of microcontroller unit (MCU) that is a small computer on a single integrated circuit that contains a processor core, memory, and programmable I/O peripherals. It is widely used due to its cheapness and low power consumption. Generally, it is of RISC architecture with many registers and limited bus size ( $8-32$ bits) ${ }^{7}$. ATmega-128 microcontroller is a high performance embedded microcontroller with reduced instruction set collection (RISC) and FLASH memory technology. It has lots of advantages which include a power consumption, fast operation, peripheral expansion capability and so on.

In addition, the ATmega-128 microcontroller also has an integrated $\mathrm{A} / \mathrm{D}$ converter module and the function of set watchdog. This microcontroller has a $128 \mathrm{~KB}$ code and $4 \mathrm{~KB}$ of data memory. It can be run with $16 \mathrm{MHz}$ crystal frequency up to 16 MIPS. It has 32 general-purpose registers, four timer/counter units, seven 8-bit input /output ports, two UART ports and can execute most of the commands in a single clock cycle. Since it is low-cost and has low power, it is widely preferred in various embedded system applications $^{8}$.

The ATmega-128 microcontroller has been connected with several supporting sensors, namely the temperature and humidity sensor (SHT-11) in the heating reactor is connected using SPI (Serial Peripheral Interface) protocol, the temperature sensor DS-1820 for measuring the sample is connected using 1-wire

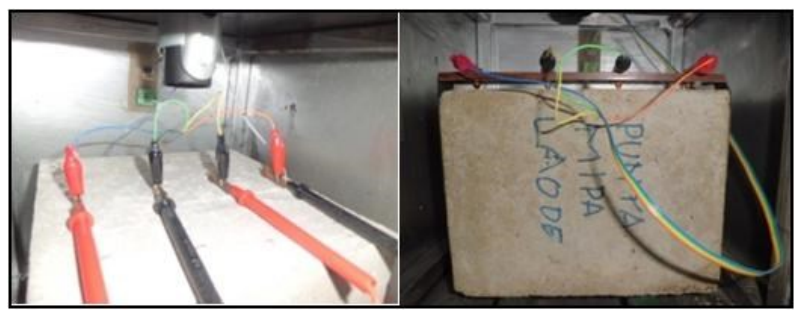

Fig. 5. Four-electrode Method Configuration of Electrical Resistivity Measuring surface (right) and the interface (left)

protocol, while the analog electrical current sensor ACS-172 is connected to the 10-bit ADC port of the 
microcontroller. The system s also equipped with RTC (Real-Time Clock) DS-1307 unit that is connected using I2C protocol. This unit is used to control the duration as well as the schedule of the characterization process in the real time mode. The data communication standard from embedded systems to the computer interfacing using a standard RS-232 serial com munication dualchannel method USART (Universal Synchronous Asynchronous Receiver Transmitter). In order to make the system more user-friendly, it is equipped with a control panel that consists of 20x4 LCD (Liquid Crystal Display), Matrix Keypad 4x4.

Table 1. Configuration of AVR ATmega-128 Pin for Embedded System to Resistivity Analyzer

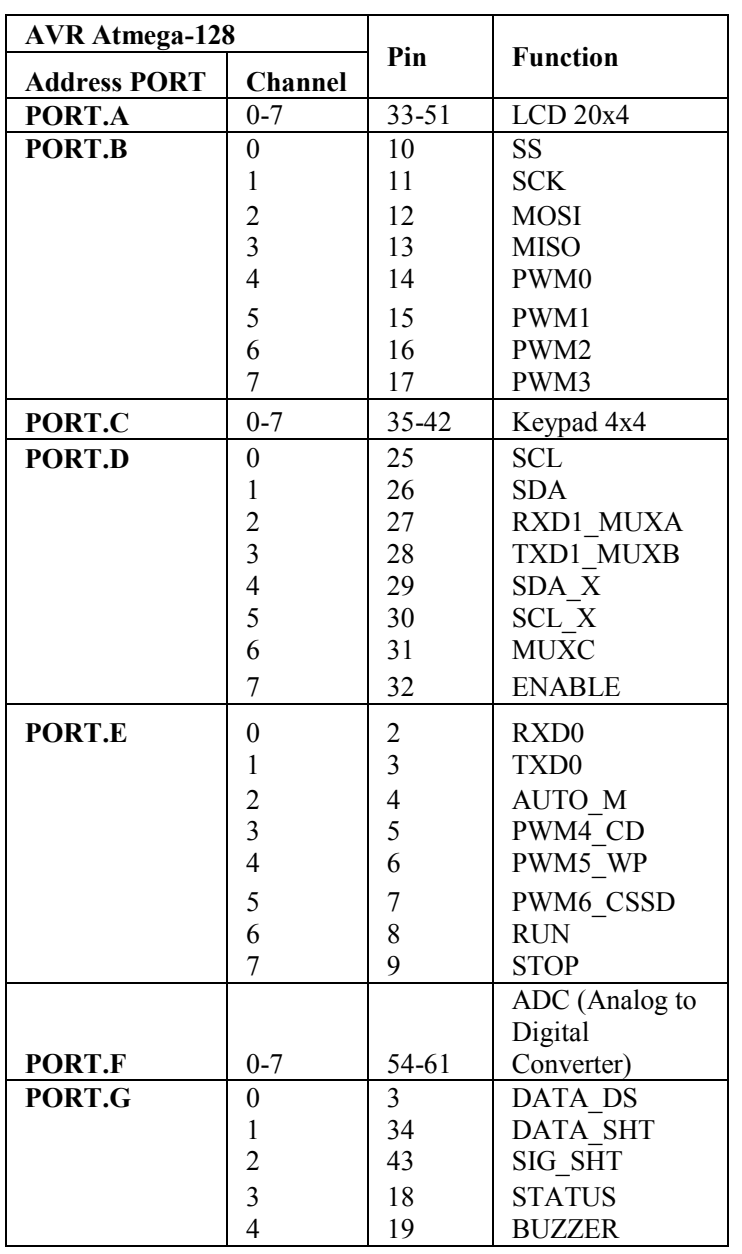

\section{The Design of The System and Its Operating Principles}

As it has been described in the previous part, the system consists of: variable power supply for generating electric current injected to the sample, an electric heater, temperature and humidity sensors, electrical current sensor. Their operating principles will be described in the following parts:

\subsection{Variable Power Supply injected to the sample}

The voltage to be injected to the sample material is generated by a variable power supply which is controlled by the AVR ATMega-128 using a PWM method. The magnitude of the voltage generated is proportional to the duty cycle of the PWM signal generated through PORTB.5 of the when duty cycle of the PWM signal is 0 , the magnitude of the voltage generated by this unit is 0 volt and when duty cycle of the PWM signal is $100 \%$. The unit generates 30 volt (the maximum value). The PWM signal has frequency 125 $\mathrm{kHz}$, and its duty cycle is in 10-bit mode.
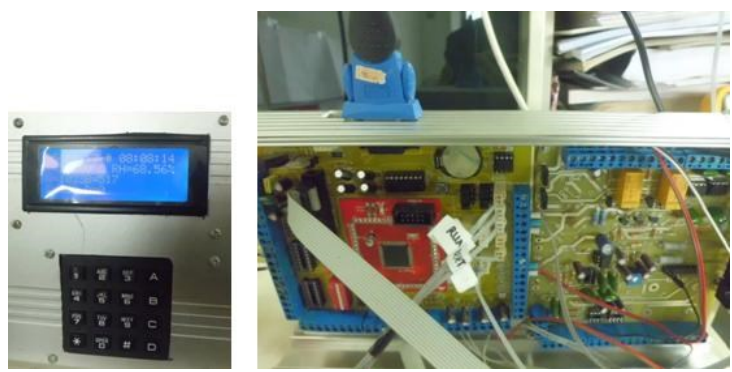

Fig. 6. Embedded System based microcontroller AVR ${ }^{\circledR}$ ATmega128 ( ${ }^{\circledR}$ and Panel Systems Electronics (right), and LCD+Keypad Matrix 4x4 Panel (left).

\subsection{System of Timer RTC (Real Time Clock) DS- 1307}

The RTC unit is connected to the microcontroller using I2C protocol, through PORTD.4 (SDA) and PORTD.5 (SCL) of the microcontroller. This unit uses $32 \mathrm{kHz}$ crystal oscillator as its clock generator. This unit is used to give information about the date and the time as well as the duration of the conducted experiment. 


\subsection{Electric Heater}

The electric heater is used to control the temperature of the heating reactor of the sample. It is controlled by the microcontroller using an ON/OFF control method via a relay which is connected to PORTB.6 as shown in figure.

\subsection{Temperature Sensor DS-1820}

DS-1820 sensor serves as a temperature measuring unit of the sample. This sensor is a digital temperature sensor which is connected using 1-wire communication standard, and is connected via PORTG.0 of the microcontroller. The sensors has been measure the temperature in range $-55^{\circ} \mathrm{C}$ to $+125^{\circ} \mathrm{C}$ at $0.5^{\circ} \mathrm{C}$. The system will be read the temperature value in 9-bit data format, and the conversion of data process in a digital format that takes $200 \mathrm{~ms}^{9}$.

\subsection{Temperature and Humidity Sensor SHT-11}

The sensor is used to measure the temperature and the humidity of the heating reactor. This unit communicates with the microcontroller using Serial Peripheral Interface (SPI) protocol through PORTG.1 and PORTG.2.

\subsection{The Electrical Current Sensor ACS-172}

The electric current flows through the sample is sensed by Hall-Effect-based current sensor ACS-172. The output voltage generated by this sensor, which is proportional to the flow current, is measured by the microcontroller through its ADC unit channel (PORTF.0). The sensitivity of the electric current in the sensor readings are around 66 to $185 \mathrm{mV} / \mathrm{A}$, with impedance of $1.8 \mathrm{~m} \Omega$ internal conductor. The sensor also has a nearly zero magnetic hysteresis character (no hysteresis curve of magnetization system) on symptoms Hall Effect of electric current measurement principle. On the sensor Total Error output of $1.5 \%$ on the testing temperature at $\mathrm{TA}=25^{\circ} \mathrm{C}$ for flow measurement error ${ }^{10}$.

\subsection{Algorithm of Program to Embedded Systems ATmega-128}

The program code which is level open to drive the microcontroller works as shown in flowchart in figure 8 . In the first step all variables used in the program are declared and all unit such as ADC, PWM, 1-wire Port, I2C Port, SPI Port, Asynchronous Serial

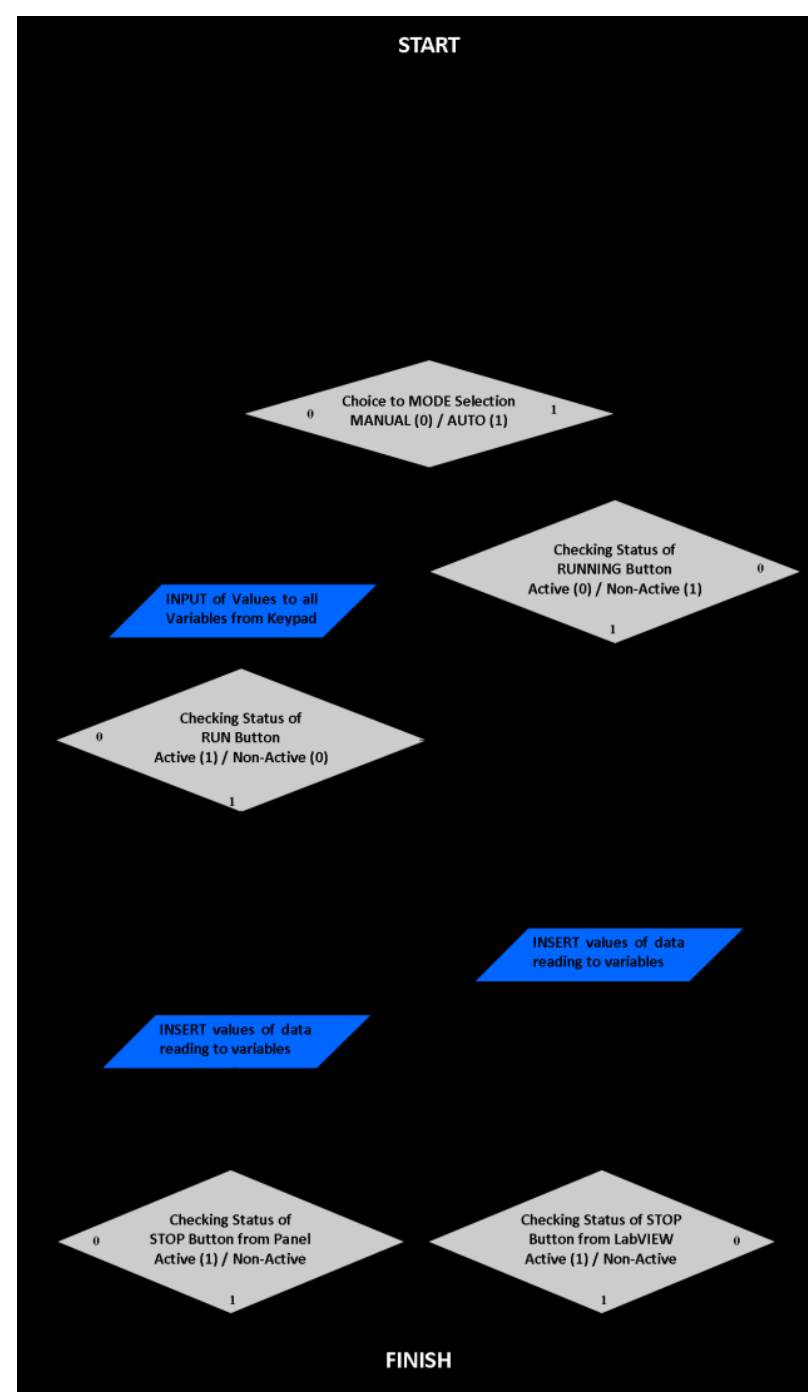

Fig. 7. General Flow-chart of embedded system

Communication Port, Keypad, as well as LCD are configured. Subsequently, it activates the SHT-11 sensor for measuring the temperature and humidity of the heating reactor, as well as activates the reading process of the RTC-1307. In the second step, the system program will detect the selected mode (automatic or manual mode) chosen by the user LCD are configured. Subsequently, it activates the SHT-11 sensor for measuring the temperature and humidity of the heating reactor, as well as activates the reading process of the RTC-1307. In the second step, the system program will detect the selected mode (automatic or manual mode) chosen by the user.

If manual mode is selected, the system scans the input data from the keypad, and then it will enter in 
running mode: or non-active mode depend on the input data entered by the user. In the next step, it will set the set-point values for all the parameters to be measure, and then reads from all the channels (1-wire, SPI, I2C, ADC), and calculating all the needed parameters. The calculated results will be displayed on LCD panel, and in the further step, the system will check the state of Stop Button, if the state of the button is active, it will stop the program, however if the stop button state is non-active, the system will still in a running mode.

If automatic mode is selected, the system will check the state of the Run Button. If the run button state is active, in the next step, the automatic-mode system will accept the set-point data input via interface panel. The system can accept a set of set-point values from the application panel as shown in Figure 10, and then the system will read from all the channels (1-wire, SPI, I2C, $\mathrm{ADC})$, and calculate all the needed variable that appropriate with the resistivity formulation. Next step, the system will display all the results on LCD panel, and all the data from calculation is sent to the Personal Computer and plotted to LabVIEW panel display as shown in Figure 9. As described in manual mode, The system program will also check the state of Stop Button. the state of the button is active, it will stop the program, however if the stop button state is non-active, the system will still in a running mode.

The data will be converted into the form of a USB to serial converter. The data conversion mechanism with MAX-232 and embedded system based on AVR microcontroller ATmega-128, as well as a USB to Serial converter cable. The hardware, system interface will received of data from the USB port with VISA techniques for read-write data communications systems in both directions (full duplex). The system of interfacing with a computer to communicate between the transmitter and receiver (computers with embedded systems).

Application of instrumentation system from VISA standard serial communication to develop on LabVIEW, and system based on serial protocol to running of measure of electrical resistivity of conductive concrete material (consists of Front Panel and Block Diagram). The front panel display consists of a GUI (Graphical User Interface) that will be displayed the Tab menu bar: Set Point, Result of Measure, Graph, Settings (communication standard between computer with embedded system/hardware), Data Table, The Result Plot, Plot Data, and Real-time Data.

The Instrumentation Experiment of Results

The Result of Measure (fig.9), this section is a viewer panel from data of measurement in characterization process. The data will be displayed to the panel Voltage input injection to sample of material (volts), Material Load Voltage (Volt), and Electrical Current is injected into the sample (Ampere). Resistivity (Ohm.m) and conductivity, as well as information from temperature measurements of Material (Celsius), Temperature and Humidity Heater Reactor (Celsius \& $\% \mathrm{RH})$. The measurement data to work on embedded system between the sensor and other electronic elements for measure system.

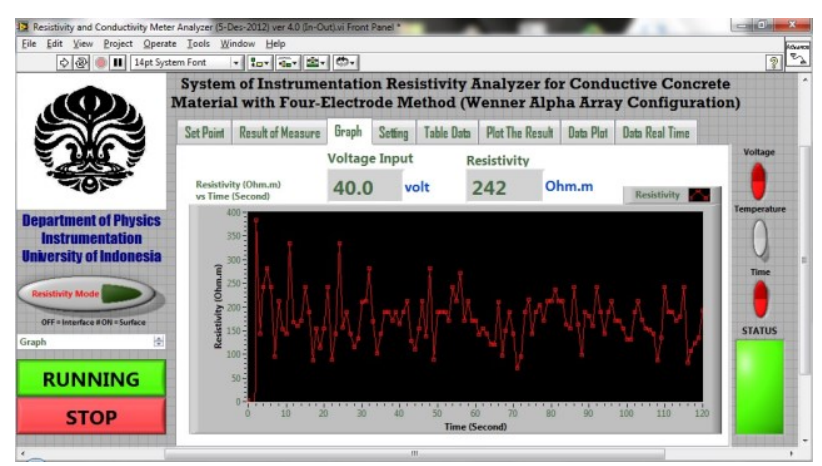

Fig. 8. The Result of Resistivity Measurement of The Conductive Concrete Material

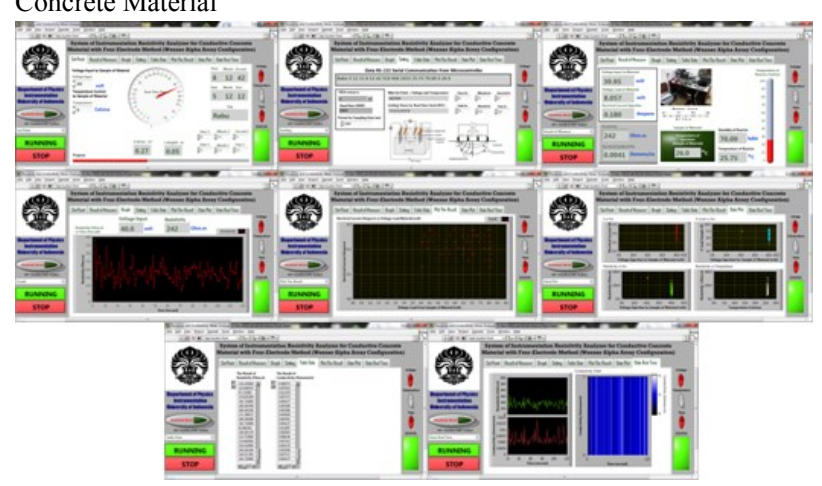

Fig. 9. Application Panel of Computer Interfacing based on LabVIEW

The Set Point is control element for value of potential difference voltage injection into the sample of material, the maximum temperature controller characterization process, as well as controlling the length of time the material characterization process. To select this panel, previously had to choose first 
characterization on the button activator Voltage, Temperature, and Time (the Front Panel at right area). The "Data Table", which is an element of the data acquisition process measurement results of material characterization. The values of data shown in this panel to measurement result data of the value of resistivity (Ohm.m) and Conductivity (Siemens/m).

The data result of characterization process will be enquired for the material standardization of electrical conductivity level with influence from physical parameters (temperature/humidity, voltage injection, time of characterization). This system of instrumentation based on embedded-system technology will be simplified of the characterization process with real-time process (data of result can be saved from PC panel with auto mode selection).

\section{Conclusion}

The automation of the process of measuring resistivity of the conductive concrete material has been successfully implemented with a microcontroller-based embedded system. The system has been integrated with a PWM-based controllable power supply, several sensors for measuring a set of physical parameters associated with the electrical resistivity of the sample and also integrated with an RTC unit for providing the timing of execution, therefore the process of the material characterization can be executed in real time. The results of the measurements can be displayed on the LCD unit as well as can be send to the PC in order to display the results graphically and record of data results via LabVIEW National Instrument).

Instrumentation system can be worked to implemented a data acquisition system with serial communication via RS-232 MAX-232 conversion on an embedded system microcontroller AVR ATmega-128. The Serial data communication standard to processed between embedded system with a Personal Computer (PC) used a USB-serial converter by National Instruments LabVIEW-based applications. Standardize to implementation of serial communication using VISA read \& write on LabVIEW program. The system can respond to a given of set-point from $\mathrm{PC}$, and all data will be processed with full-duplex communication system between the computer and embedded systems.

Process characterization can be done to voltage injection variation into the material, as well as the maximum temperature variation in the heating reactor, and the variation of the length of time the material characterization process. This application can be displayed to characterization process measurement data of resistivity/conductivity material, the voltage injection, the voltage load of material, electrical current injection into the sample of material, the temperature of material, temperature and humidity in heating reactor, and time information in real-time system. The panel has designed to display a LabVIEW measurement data in numeric and graphical data plotted. The result of data characterization can be saved and printed via printer from PC.

\section{Acknowledgements}

This research was conducted in the Laboratory of Instrumentation and Control, Laboratory of Electronics, and Laboratory of Computation - Microcontroller, Department of Physics, University of Indonesia under supervised My Advisor and Supervisor (Dr. Prawito, Drs. Arief Sudarmaji, M.T, Dr. Sastra Kusuma Wijawa) was discussed and advice for my research. And significantly, many thanks to Multiferroic Research Group, Material Science, Physics, University of Indonesia (Laboratory Multiferroic: Drs. Arief Sudarmaji, Dr. Muhammad Hikam, Dr. Bambang Soegijono).

We would like thanks to Dr. Ir. Elly Tjahjono, DEA and team (Head of Structures and Materials Laboratory), Department of Civil Engineering, Faculty of Engineering, University of Indonesia, and also to her staff who made the conductive concrete material that has been tested.

The publicity of this paper partially supported by SINas Research Grant 2014 under contract no. 17/SEK/INSINAS/PPK/I/2014, Ministry of Research and Technology, the Republic of Indonesia

\section{References}

1. Siroky, D., and Steffan, P, Control Unit for Smart Concrete Used as Resistive Heating. IEEE 33rd International Seminar on Electronics Technology, 2010, pp. 45-49.

2. Karhunen, K., et al, Electrical Resistance Tomography Imaging of Concrete. Elseiver - Cement and Concrete Research-40, 2010, pp. 137-145.

3. Lataste, J. F., et al, Electrical Resistivity Measurement Applied to Cracking Assessment on Reinforced Concrete Structures in Civil Engineering. Elseiver NDT\&E International-36, 2003, pp. 383-394.

4. Morris, W, Corrotion of Reinforcing Steel Evaluated by Means of Concrete Resistivity Measurements. ElseiverPergamon-Corrosion Science-44, 2002, pp. 81-99. 
5. Lee, C. Y., and Wang, S. R, Application of Fourelectrode Method to Analysis Resistance Characteristics of Conductive Concrete. World Academy of Science, Engineering and Technology-72, 2010, pp.101-105.

6. Datasheet AVR

ATmega128:

. Younho Leea, Ill-Hee Kimb,Yongsu Parkb. Improved multi-precision squaring for low-end RISC microcontrollers. The Journal of Systems and Software86, 2013, pp.60-71.

8. Çakirolu, Murat. Software implementation and performance comparison of popular block ciphers on 8bit low-cost microcontroller. International Journal of the Physical Sciences Vol. 5 (9), 2010, pp. 1338-1343.

9. DS-1820 1-Wire Parasite-Power Digital Thermometer http://datasheets.maximintegrated.com/en/ds/DS18S20.p df

10. ACS172 Fully Integrated, Hall-Effect-Based Linear Current Sensor IC with 2.1 kVRMS Voltage Isolation and a Low-Resistance Current Conductor, http://www.allegromicro.com/ /media/Files/Datasheets/ ACS712-Datasheet.ashx 